\title{
Drug Resistance and R Plasmids Among Salmonella and Escherichia coli Isolated from Broiler Chickens
}

\author{
-A Comparison between the Incidence in \\ $1974 \sim 1975$ and in $1978 \sim 1979$
}

(Received May 6, 1980)

\begin{abstract}
Kiyoshi TAGaWA
(Department of Food Science, Faculty of Agriculture, Kagawa University; Miki-cho, Kita-gun, Kagawa)
\end{abstract}

\begin{abstract}
Salmonella and $E$. coli strains, isolated from broiler chickens over the periods May 1974 to March 1975 (Period I) and May 1978 to March 1979 (Period II), were investigated and compared with respect to drug resistance.

In period I, $98 \%$ of the Salmonella strains and all of the E. coli strains were resistant to one or more drugs, while in period II, $75 \%$ of the former and $98.1 \%$ of the latter were resistant to at least one drug. The frequencies $(\%)$ of the individual drug resistances in the Salmonella strains were as follows (figures for period II in parentheses): tetracycline 66 (17), streptomycin 43 (16), sulfisoxazole 84 (59), chloramphenicol 13 (0), kanamycin 15 (38), ampicillin $10(8)$, cephaloridine $14(11)$, gentamicin $3(3)$, furatrizine 90 (37), nalidixic acid 2 (0), and those in the $E$. coli strains were: tetracycline $93.3(85.3)$, streptomycin 69.8 (65.7), sulfisoxazole 86.4 (89.4), chloramphenicol 35. 4 (25.7), kanamycin 47.0 (52.8), ampicillin 7. 4 (22.2), cephaloridine $5.7(19.0)$, gentamicin $3.6(0.9)$, furatrizine $93.8(77.3)$, nalidixic acid $1.0(0.7)$.

These results are of interest in relation to the amended law concerning feed additives enacted in 1975 . However, the increasing frequencies of kanamycin and ampicillin resistances together with the prevalence of $\mathrm{R}$ plasmids among the $E$. coli strains suggest that it will be difficult to prevent the spread of drug-resistant bacteria in broiler rearing.
\end{abstract}

Key words: drug resistance; R plasmids; Salmonella; Escherichia coli; broiler chicken; antibiotics; feed additives; kanamycin; ampicillin; furatrizine

\section{Introduction}

The drastic increase in drug-resistant bacteria accompanying the use of antimicrobial agents in animal husbandry has posed serious problems in food hygiene and public health, because in the majority of cases their resistance is plasmid-mediated and transferable from non-pathogens to pathogens or vice versa.

A recent trend is to limit the use of such drugs in animal husbandry, and provisional guidelines to control such use were established in the Swann Committee's recommendations 'on the use of antibiotics in animal husbandry and veterinary medicine', issued in England in $1969^{11}$.

In Japan, an amendment of the law on provision of feed additives ${ }^{2)}$, which was designed to limit the use of antimicrobial agents in animal husbandry more strictly than was previously the case, was enacted in 1975 and came into force in July 1976.

In contrast to the abundance of information on the development of drug resistance and $\mathrm{R}$ plasmids in bacteria isolated from animals in relation to the use of drugs in animal feeds, reports concerning the effects of cessation of the use of drugs in the feeds on the prevalence of drug-resistant bacteria in animals are scarce. Larsen and Neilsen ${ }^{3)}$ reported that coliforms isolated from swine 
Table 1. Isolation Frequency of Drug-resistant Salmonella Strains

\begin{tabular}{|c|c|c|c|}
\hline & & Period I & Period II \\
\hline & & No. of strains $(\%)$ & No. of strains $(\%)$ \\
\hline Suscept & & $2(2)$ & $16(25)$ \\
\hline Resista & 1 drug & $8(9)$ & $14(22)$ \\
\hline " & 2 drugs & $19(21)$ & $19(30)$ \\
\hline$"$ & $3 "$ & $21(23)$ & $2(3)$ \\
\hline " & $4 \prime \prime$ & $18(20)$ & $2(3)$ \\
\hline$"$ & $5 \prime \prime$ & $15(16)$ & $8(13)$ \\
\hline " & $6 \prime \prime$ & $6(7)$ & $1(2)$ \\
\hline$"$ & $7 \prime \prime$ & $2(2)$ & $1(2)$ \\
\hline$"$ & $8 \prime \prime$ & $1(1)$ & $0(0)$ \\
\hline & & $92(100)$ & $63(100)$ \\
\hline
\end{tabular}

herds have exhibited a sharp drop in multiple resistance after restriction of the use of tetracycline and penicillin in their feeds. However, Smith ${ }^{4}$ found that the incidence of strains resistant to tetracycline decreased only slightly in the 4 years following implementation of the Swann Committee's recommendations. Linton $^{5)}$ more recently concluded that there has been little adherence to the recommendations of the Swann Committee. The present study has been undertaken to assess the incidence of drug resistance and $\mathrm{R}$ plasmids among Salmonella and Escherichia coli strains isolated from broiler chickens. Attention has been directed especially to evaluating the extent to which the restricted use of drugs in broiler rearing has affected the incidence or pattern of drug resistance and $\mathrm{R}$ plasmids.

\section{Materials and Methods}

\section{Isolation of bacteria}

Fresh feces samples were collected from broiler chickens on 20 different farms situated in Kagawa.

To isolate Salmonella strains, samples were plated on a brilliant green agar (Nissui Pharmaceutical Co.) after enrichment in Rappaport medium (Eiken Chemicals Co.). The candidate colonies were streaked on triple sugar iron agar (Nissui Pharmaceutical Co.) slants after two successive single-colony isolations. These isolates were examined biochemically and serologically for O-antigen with sera representing six Salmonella O-groups
(Kitasato Institute).

For the isolation of $E$. coli strains from the same samples, pre-enrichment was carried out with a brilliant green lactose bouillon broth (Nissui Pharmaceutical Co.) with subsequent plating on an eosin methylene blue agar (Eiken Chemicals Co.). Up to three isolates per sample were subsequently identified by the methods outlined by Edwards and Ewing ${ }^{6)}$.

\section{Drugs}

Tetracycline (TC) was purchased from Sigma Chemical Co., London; streptomycin (SM) from Takeda Chemical Industries, Osaka; sulfisoxazole (SA) from Yamanouchi Pharmaceutical Co., Tokyo; chloramphenicol (CM) from Sankyo Co., Tokyo; kanamycin (KM) from Meiji-Seika Co., Tokyo; ampicillin(APC) from Banyu Pharmaceutical Co., Tokyo; gentamicin (GM) from Schering Corporation, Kenilworth; and nalidixic acid (NA) from Daiichi Pharmaceutical Co., Tokyo. Cephaloridine (CER) and furatrizine (FT) were gifts from Torii Chemicals, Tokyo, and Toyama Chemicals Industries, Tokyo, respectively.

\section{Test for drug resistance}

Drug resistance of strains isolated was determined by the agar dilution method recommended by the Japan Chemotherapeutic Association $^{7)}$. One hundred-fold dilutions of overnight cultures were spotted on brain heart infusion agar (Difco Laboratories)plates containing one of the test drugs with the exception of SA and incubated at $37^{\circ} \mathrm{C}$ for 
Table 2. Isolation Frequency of Drug-resistant E. coli Strains

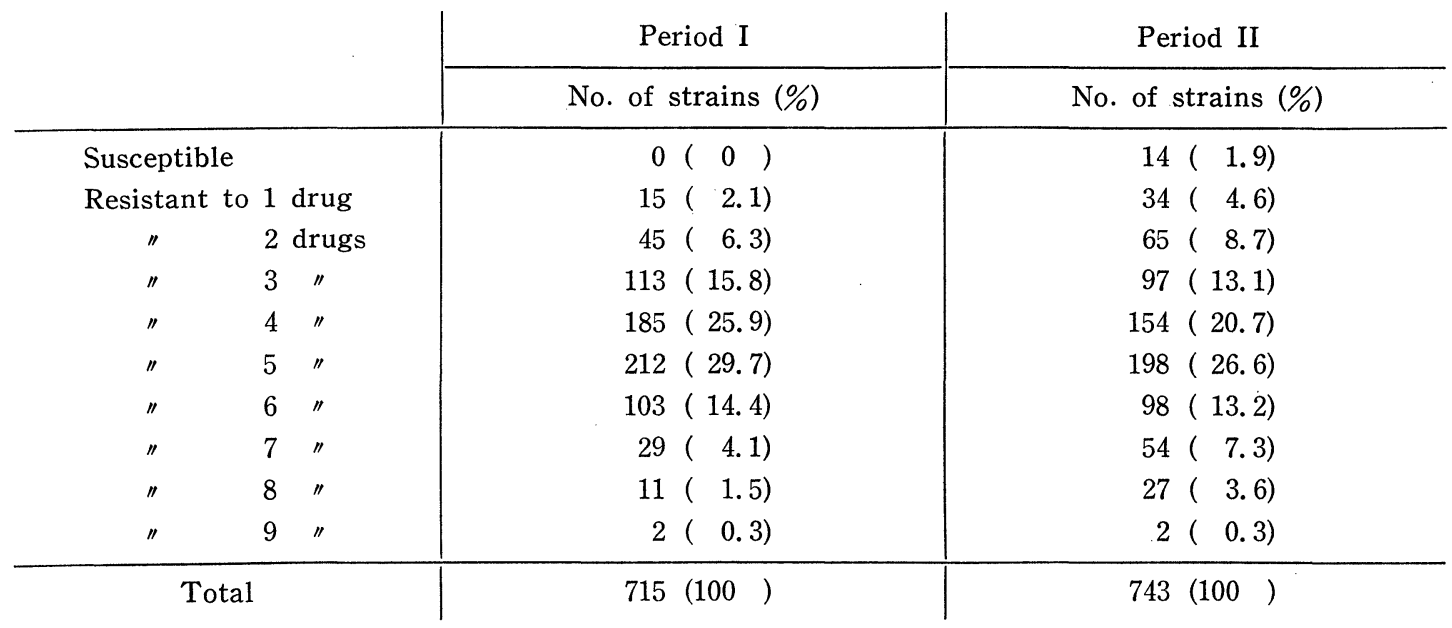

$24 \mathrm{hr}$. For testing SA resistance, semisynthetic medium A agar ${ }^{8)}$ plates containing this drug were used. A strain was considered to be resistant if growth occurred on the plates containing the following concentrations of drugs: TC, 12.5; SM, 25; SA, 200; CM, 12.5; KM, 6. 25; APC, 12.5; CER, 6. 25; GM, 3.1; F $\mathrm{T}, 1.6 ; \mathrm{NA}, 25$ (the concentrations are given in $\mu \mathrm{g} / \mathrm{ml})$.

Test for $R$ plasmids

The strains found to be sensitive to NA and resistant to one or more of the other drugs were examined for transmissible drug resistance. The recipient strain used was NAresistant $E$. coli ML1410, kindly supplied by Prof. S. Mitsuhashi, Gunma University. Equal volumes of overnight broth cultures of the prospective donor and the recipient were mixed and incubated at $37^{\circ} \mathrm{C}$ for $18 \mathrm{hr}$. The mixed cultures were streaked on brain heart infusion agar or semi-synthetic medium A agar plates containing $25 \mu \mathrm{g} / \mathrm{ml}$ of NA together with a single appropriate drug. After $24 \mathrm{hr}$ of incubation at $37^{\circ} \mathrm{C}$, colonies were picked from the plates and subjected to three successive single-colony isolations on a medium of the same constitution. The drug resistance patterns of all colonies thus obtained were determined by the replica plating method, as described previously ${ }^{9)}$.

\section{Results}

In the experiments carried out from May
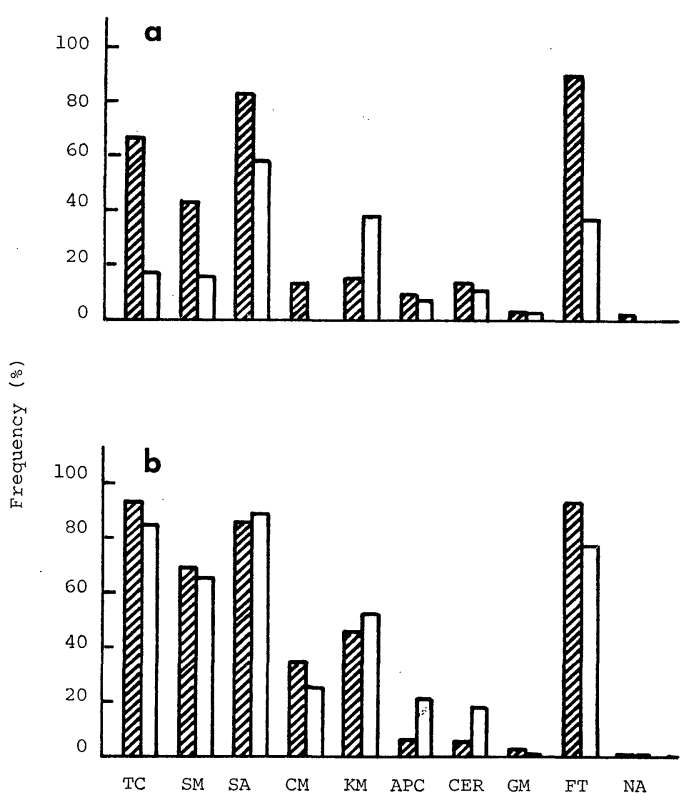

Fig. 1. The frequencies of individual drug resistance among Salmonella (a) and $E$. coli (b) strains isolated in periods I and II (the hatched columns indicate the isolates in period I)

1974 to March 1975 (Period I), 90 of 92 Salmonella isolates and all $E$. coli isolates were resistant to one or more of the antimicrobial agents tested, while in the period from May 1978 to March 1979 (Period II), 47 of 63 Salmonella isolates and 729 of $743 \mathrm{E}$. coli were resistant to at least one drug. As 
Table 3. Incidence and Transferability of Resistance Patterns among Salmonella Strains

\begin{tabular}{l|c|c|c|r|r|r|r}
\multicolumn{2}{c|}{ Period I } & \multicolumn{4}{c}{ Period II } \\
\hline Resistance pattern & $\begin{array}{c}\text { No. of } \\
\text { strains }\end{array}$ & $\begin{array}{c}\text { No. of } \\
\text { strains } \\
\text { transferred }\end{array}$ & $\begin{array}{c}\text { Percent } \\
\text { transfer }\end{array}$ & Resistance pattern & $\begin{array}{c}\text { No. of } \\
\text { strains }\end{array}$ & $\begin{array}{c}\text { No. of } \\
\text { strains } \\
\text { transferred }\end{array}$ & $\begin{array}{c}\text { Percent } \\
\text { transfer }\end{array}$ \\
\hline TC. SA. FT & 16 & 3 & 19 & SA. KM & 13 & 5 & 39 \\
TC. SM. SA. FT & 12 & 7 & 58 & SA & 6 & 0 & 0 \\
SA. FT & 11 & 2 & 18 & FT & 6 & 0 & 0 \\
TC. SM. SA. CM. FT & 6 & 3 & 50 & TC.SM.SA. KM. FT & 5 & 5 & 100 \\
TC. FT & 5 & 1 & 20 & SA.FT & 5 & 0 & 0 \\
FT & 5 & 0 & 0 & Others & & 7 & 58 \\
TC. SM.SA. KM. FT & 4 & 2 & 50 & & & &
\end{tabular}

a) NA-resistant strains were not tested.

Table 4. Incidence and Transferability of Resistance Patterns among E. coli Strains

\begin{tabular}{|c|c|c|c|c|c|c|c|}
\hline \multicolumn{4}{|c|}{ Period I } & \multicolumn{4}{|c|}{ Period II } \\
\hline Resistance pattern & $\begin{array}{l}\text { No. of } \\
\text { strains }\end{array}$ & \begin{tabular}{|c|} 
No. of \\
strains \\
transferred
\end{tabular} & $\begin{array}{l}\text { Percent } \\
\text { transfer }\end{array}$ & Resistance pattern & $\begin{array}{l}\text { No. of } \\
\text { strains }\end{array}$ & \begin{tabular}{|c|} 
No. of \\
strains \\
transferred
\end{tabular} & $\begin{array}{l}\text { Percent } \\
\text { transfer }^{a)}\end{array}$ \\
\hline TC.SM.SA.FT & 116 & 79 & 68.1 & TC.SM.SA. KM.FT & 96 & 67 & 69.8 \\
\hline TC. SM.SA. KM. FT & 73 & 53 & 72.6 & TC.SM.SA. FT & 66 & 43 & 65.1 \\
\hline TC.SM.SA. CM. FT & 71 & 45 & 63.4 & TC.SA. FT & 54 & 12 & 22.2 \\
\hline TC.SA.FT & 59 & 18 & 30.5 & TC.SM. SA. CM. KM. & 51 & 38 & 74.5 \\
\hline $\begin{array}{l}\text { TC. SM. SA. CM. KM. } \\
\text { FT }\end{array}$ & 42 & 31 & 73.8 & $\begin{array}{l}\text { FT } \\
\text { TC. SM. KM. APC. }\end{array}$ & 46 & 40 & 87.0 \\
\hline TC.SA.CM. FT & 23 & 8 & 34.8 & (CER). & . & & \\
\hline TC. FT & 23 & 4 & 17.4 & $\begin{array}{l}\text { TC. SM. SA. CM. KM. } \\
\text { APC. (CER). FT }\end{array}$ & 28 & 28 & 100.0 \\
\hline TC.SA. KM. FT & 19 & 11 & 57.9 & TC. SA. KM. FT & 26 & 13 & 50.0 \\
\hline TC.SM.FT & 18 & 6 & 33.3 & TC.SM.SA & 24 & 8 & 33.3 \\
\hline Others & 271 & 176 & 66.7 & TC.SM. SA. CM. FT & 16 & 9 & 56.3 \\
\hline & & & & $\begin{array}{l}\text { TC. SM.SA. APC. } \\
{\text { (CER })^{\mathrm{b})}}^{\text {S. }}\end{array}$ & 16 & 12 & 75.0 \\
\hline & & & & Others & 306 & 203 & 67.4 \\
\hline
\end{tabular}

a) NA-resistant strains were not tested.

b) Not all strains were resistant to CER.

shown in Tables 1 and 2, most strains were multiply resistant, and singly resistant strains were scarce among both Salmonella and $E$. coli isolates.

On comparing the incidence of multiply resistant strains in period $I$ to that in period II, the proportion of Salmonella strains resistant to three or more drugs decreased by about one-third from period I to period II, whereas no significant change in the incidence of multiply resistant strains was ob. served among $E$. coli isolates.

The incidence of the strains resistant to individual drugs among Salmonella and E. coli isolates for the two periods is shown in Fig. 1. In period $I$, a high percentage of strains resistant to TC, SM, SA and FT was observed in both bacterial strains. However, in period II, the incidence of resistance to these drugs decreased significantly among Salmonella strains, but only slightly in $E$. coli strains except for resistance to SA (which increased 
Table 5. Transferability of Individual Drug Resistance Determinants among Salmonella Strains

\begin{tabular}{c|c|c|c|c} 
& \multicolumn{2}{|c|}{ Period I } & \multicolumn{2}{|c}{ Period II } \\
\cline { 2 - 5 } Resistance determinant & $\frac{\text { No. of strains transferred }}{\text { No. of strains tested }}$ & Percent & $\frac{\text { No. of strains transferred }}{\text { No. of strains tested }}$ & Percent \\
\cline { 2 - 5 } TC & $28 / 59$ & 47 & $6 / 11$ & 55 \\
SM & $26 / 39$ & 67 & $9 / 10$ & 90 \\
SA & $23 / 76$ & 30 & $12 / 37$ & 32 \\
CM & $3 / 11$ & 27 & 0 & 46 \\
KM & $6 / 13$ & 46 & $11 / 24$ & 100 \\
APC & $7 / 9$ & 78 & $5 / 5$ & 71 \\
CER & $10 / 13$ & 77 & $5 / 7$ & 0 \\
GM & $2 / 3$ & 67 & $0 / 2$ & 22
\end{tabular}

Table 6. Transferability of Individual Drug Resistance Determinants among E. coli Strains

\begin{tabular}{c|c|c|c|c}
\multirow{2}{*}{ Resistance determinant } & \multicolumn{2}{|c|}{ Period I } & \multicolumn{2}{c}{ Period II } \\
\cline { 2 - 5 } & $\frac{\text { No. of strains transferred }}{\text { No. of strains tested }}$ & Percent & $\frac{\text { No. of strains transferred }}{\text { No. of strains tested }}$ & Percent \\
\hline TC & $320 / 661$ & 48.4 & $329 / 630$ & 52.2 \\
SM & $258 / 493$ & 52.3 & $289 / 484$ & 59.7 \\
SA & $238 / 611$ & 39.0 & $271 / 660$ & 41.1 \\
CM & $107 / 250$ & 42.8 & $89 / 188$ & 47.3 \\
KM & $227 / 332$ & 68.4 & $242 / 388$ & 62.4 \\
APC & $43 / 52$ & 82.7 & $121 / 162$ & 74.7 \\
CER & $32 / 40$ & 80.0 & $116 / 139$ & 83.5 \\
GM & $10 / 24$ & 41.7 & 6 & 33.3 \\
FT & $9 / 664$ & 1.4 & $13 / 571$ & 2.3
\end{tabular}

in period II). Decreased incidence of resistance to CM was also observed for both genera of bacteria, and no Salmonella strain with this resistance was observed during period II.

In contrast, the incidence of $\mathrm{KM}$ resistance among Salmonella and $E$. coli isolates and that of APC and CER resistance among $E$. coli isolates increased significantly in period II. The incidence of resistance to GM and NA was very low for both genera throughout period I and period II.

Resistance patterns occurring in Salmonella strains fell into 28 and 15 different types in period I and period II, respectively. On the other hand, $E$. coli strains in period I had a total of 82 different resistance patterns, and the resistant $E$. coli strains in period II had 84 different patterns. The commonly occurring resistance patterns together with frequencies of their transferability to the recipient $E$. coli ML1410 among the Salmonel$l a$ and $E$. coli strains are shown in Tables 3 and 4, respectively. Among Salmonella strains, TC.SA.FT, TC.SM.SA.FT and SA.FT resistance patterns were most frequent in period I (43\% of the resistant strains), and SA.KM and SA only or FT only resistance patterns were most common in period II $(53 \%$ of the resistant strains). On the other hand, in $E$. coli strains, there was a marked similarity between the frequently occurring resistance patterns in period I and period II; TC.SM.SA.FT, TC.SM.SA.KM.FT, TC.SA.FT and TC.SM.SA.CM.KM.FT patterns were the 
most frequent, accounting for $40.6 \%$ and 35.9 $\%$ of the strains isolated in period $\mathrm{I}$ and period II, respectively, and in addition to these resistance patterns, the TC.SM.SA.CM. FT pattern also occurred frequently in period $I$ and the TC.SM.KM.APC. (CER). FT pattern in Period II.

Among Salmonella isolates tested for transferability of drug resistance determinants, 37 $(42 \%)$ of the strains in period I and $17(36 \%)$ of the strains in period II were capable of transferring one or more of the drug resistances, while in the $E$. coli isolates, $431(60.9$ $\%$ ) of the strains in period I and $473(65.3 \%)$ of those in period II were found to transfer at least one of their resistance determinants.

There was a significant difference between the transferability of the singly or doubly resistant strains and that of the strains resistant to three or more drugs; among Salmonella isolates, of the total of 60 strains resistant to one or two drugs, only $9(15 \%)$ possessed $\mathrm{R}$ plasmids, and among $E$. coli isolates tested, $35(22.3 \%)$ of the 157 strains having resistance to one or two drugs carried $\mathrm{R}$ plasmids.

The transferability of the different resistance determinants among the Salmonella and $E$. coli isolates for the two periods is shown in Tables 5 and 6 , respectively. In both the Salmonella and E. coli strains, APC and CER resistances were highly transmissible as compared to the transfer of resistance to the other drugs. Furthermore, of a total of 159 strains tested which showed resistance to both drugs, $128(80.5 \%)$ were capable of transferring APC and CER resistance simultaneously to the recipient strain. In contrast, the FT resistance determinant was rarely transferable among Salmonella or $E$. coli strains. Although most of the strains were resistant to FT, only 5 strains of Salmonella and 22 strains of $E$. coli isolates transferred this resistance to the recipient.

\section{Discussion}

The significantly lower incidence of drugresistant strains isolated in period II than period I is probably a consequence of the restricted use of antimicrobial agents in broiler rearing under the amended legislation on feed additives during period II.
A precise comparison of the frequency of drug resistance of Salmonella isolates with that of $E$. coli isolates cannot be made due to the small number of Salmonella strains isolated. However, in general Salmonella strains were less resistant than $E$. coli strains and the proportion of multiply resistant strains was lower than that of the $E$. coli strains, especially among isolates from period II. One possible explanation is that plasmids conferring resistance to some drugs are more unstable in Salmonella strains than in E. coli strains and are easily lost from Salmonella in the absence of selective pressure from these drugs, as reported by Hashimoto et $a l .{ }^{10)}$ and Watanabe et $a l .{ }^{11)}$ An alternative explanation is that $E$. coli is a component of the resident flora of the gut of chickens, where it is exposed for long periods to the various drugs. On the other hand, Salmonella only occasionally infects chickens (probably being associated with some poultry diseases), and the duration of selection for resistant strains is less than in the case of $E$. coli, so that $E$. coli is more resistant than Salmonella.

In contrast to the decreased incidence of resistance to $\mathrm{TC}, \mathrm{SM}$ and $\mathrm{FT}$ in period II compared to that in period I, the increased incidence of $\mathrm{KM}$ resistance is probably a reflection of the increasing usage of this drug in broiler rearing in period II. Similar results on KM resistance have also been reported for Salmonella and E. coli by Nakaya et $a l{ }^{12)}$ and Suzuki ${ }^{13)}$, respectively. Moreover, the increased incidence of APC resistance among the $E$. coli isolates in period II is presumably a result of the prevailing use of this drug as a therapeutic drug, since penicillin, which possesses the same $\beta$-lactam configuration as APC, was prohibited for use in broiler rearing as a feed additive by the 1975 legislation. The use of APC was also probably largely responsible for the presence of strains resistant to CER, which has not been permitted for use in animal husbandry, because of its susceptibility to $\beta$-lactamases. Among the several kinds of $\mathrm{R}$ plasmid-mediated $\beta$-lactamase known, TEM type $\beta$-lactamase is the most common in gram-negative bacteria and has strong activity against both 
$\mathrm{APC}$ and $\mathrm{CER}^{14 \sim 16)}$. The present results suggested that many of the strains resistant to CER elaborate a TEM-type enzyme in view of the fact that they are also resistant to APC and cotransfer CER and APC resistance. It was surprising that a large number of strains isolated in period II were resistant to FT, even though all nitrofuran compounds were prohibited for use in animal husbandry during that period and $\mathrm{R}$ plasmids conferring FT resistance are scarce. As regards resistance to nitrofuran, McCalla et al. ${ }^{17)}$ have shown that in the acquisition of nitrofuran resistance by bacteria, two mutational steps are involved; one is a partial loss of nitroreductase activity and the second is loss of the remaining activity. On the other hand, Arai ${ }^{18)}$ reported that in a few $E$. coli strains, a moderate resistance to nitrofuran derivatives was mediated by an $\mathrm{R}$ plasmid.

The prevalence of $\mathrm{R}$ plasmid-bearing strains among $E$. coli means that it is difficult to prevent the spread of drug-resistant bacteria by simply withdrawing drugs from use in broiler rearing. However, restriction of the use of drugs seems to be effective for suppressing the acquisition of drug resistance by incoming bacteria such as Salmonella, as reported here.

A continuing survey on the incidence of drug-resistant bacteria and $\mathrm{R}$ plasmids in broiler rearing will be important in evaluating adherence to the 1975 legislation relating to feed additives.

\section{References}

1) Swann, M. M.: "Report of Joint Committee on the Use of Antibiotics in Animal Husbandry and Veterinary Medicine", Her Majesty's Sta. tionery Office, London (1969).
2) Prime Minister's Office: "Law on the Safety Assureance and Quality Improvement of Feeds" (Law No. 68), Japan Gazette, No. 14568 (1975).

3) Larsen, J., Neilsen, N.: Nordisk Veterinacrimedicin, 27, 353 364 (1975).

4) Smith, H. W.: Nature, 258, 628 630 (1975).

5) Linton, A.: Veterinary Record, 100, 354 360 (1977).

6) Edwards, P. R., Ewing, W. H.: "Identification of Enterobacteriaceae", 3rd ed., Burgess Publishing Co., Minneapolis (1972).

7) Ogoshi, M.: Chemotheraphy, 22, 1126 1128 (1974).

8) Nagai, Y., Mitsuhashi, S.: J. Bacteriol., 109, $1 \sim 7$ (1972).

9) Tagawa, K.: Tech. Bull. Fac. Agr. Kagawa Univ., 30, 107 112 (1978).

10) Hashimoto, H., Tanaka, T., Mitsuhashi, S.: Japan J. Microbiol., 17, 331 337 (1973).

11) Watanabe, H., Ike, Y., Hashimoto, H., Mitsuhashi, S.: "Microbial Drug Resistance" Vol. 2, ed. by Mitsuhashi, S., p. 111 119 (1979), Japan Scientific Societies Press, Tokyo.

12) Nakaya, N., Horiuchi, S., Yoshida, Y., Sakai, S., Ito, T., Saito, K., Terayama, T., Zenyoji, H., Onogawa, T.: "Microbial Drug Resistance" Vol. 2, ed. by Mitsuhashi, S., p. 207 209 (1979), Japan Scientific Societies Press, Tokyo.

13) Suzuki, K., Igarashi, T.: Modern Media, 22, $237 \sim 247$ (1976)

14) Heffron, F., Sublett, R., Hedges, R. W., Jacob, A., Falkow, S.: J. Bacteriol., 122, 250 256 (1975).

15) Hedges, R. W., Datta, N., Kontomichalou, P., Smith, J. T.: ibid., 117, 56 62 (1974).

16) Matthew, M., Hedges, R.W., Smith, J.T.: ibid., 138, 657 662 (1979).

17) McCalla, D. R., Kaiser, C., Green, M. H. L.: ibid., 133, 10 16 (1978).

18) Arai, T.: "Plasmids; Medical and Theoretical Aspects" ed. by Mitsuhashi, S., Rosival, L., Krcmery, V., p. 47 52 (1977), Springer Verlag, Berlin. 